Proceedings of the 52nd Hawaii International Conference on System Sciences | 2019

\title{
Advancing Spatiotemporal Modeling of Access to Healthcare - A Methodological Perspective
}

\author{
Nasser Sharareh \\ University of Utah \\ nasser.sharareh@hsc.utah.edu
}

\author{
Nasim S. Sabounchi \\ Binghamton University \\ sabounchi@,binghamton.edu
}

\author{
Rachel Hess \\ University of Utah \\ rachel.hess@hsc.utah.edu
}

\begin{abstract}
Modelers apply system dynamics (SD) modeling in various fields for different purposes including policy analysis, however, they need to integrate $S D$ with other methodologies to facilitate the inclusion of spatial factors and study their influence on the system's behavior. We investigate the combination of SD modeling with Geographic Information Systems using healthcare data to facilitate the study of both spatial and systemic factors for more effective policy design. We propose an algorithm for integrating these methodologies and explain one of its applications in the complex health systems-Medicaid beneficiaries' access to primary care (PC). Our results reveal insights and information that were not available through merely SD modeling; this approach provides the opportunity for policymakers to learn about the influence of spatiotemporal factors on health outcomes in a complex health system, and identify the areas with a high need for PC providers.

Keywords: Spatial System Dynamics; GIS Mapping; Complex systems; Healthcare Access; Medicaid, Big-Data.
\end{abstract}

\section{Introduction}

Researchers have used system dynamics (SD) modeling in different disciplines including social sciences [1, 2], management studies [3, 4], public health studies [5, 6], and engineering [7] in order to understand the underlying feedback structure of the system and investigate the influence of different intervention scenarios on the final behavior for policy analysis purposes. SD modelers use different data sources such as behavioral, demographic, and environmental data, which facilitate the modeling process and provide useful insights about the system. However, considering spatial factors and their interactions with other variables in SD models is not straightforward. Modeling this kind of features such as distance and location of variables within the system is especially critical when it informs policy design and analysis. For instance, the spread of an infectious disease within a very small community does not involve critical spatial factors. However, in order to design interventions to improve access to primary care (PC), the location of providers and their distance from patients are considered as the primary barriers to access to care. Therefore, considering spatial factors in our analyses is vital and we need a tool to analyze these factors for the determined geographical boundary.

Geographic Information Systems (GIS) mapping has become very popular among researchers and practitioners for its strength in providing useful information about health systems, and more specifically for healthcare access. For instance, Dulin et al. [8] used GIS mapping to identify high-need regions for PC access. They studied different factors such as median household income, number of visits to emergency departments (ED), and insurance coverage to determine the PC need. Edward and Biddle [9] used geospatial analysis to assess the barriers to healthcare access among a specific group of immigrants and concluded that spatial factors including healthcare facilities' locations and transportation issues are forming the barriers to healthcare access. Garcia et al. [10] investigated the geographic access to providers for pediatric asthma. They found that health outcomes of these patients vary across different regions and it was associated with the level of access to care and other demographic variables including patients' education and income level. In order to improve the health outcomes of the pediatric asthma patients, authors suggested targeting regions that lack providers and have low-income households. Other studies have used GIS mapping to identify regions which are called hot spots, such as lack of parental clinics in areas with high-needs mother [11], or lack of healthcare providers in high-density areas [12]. In general, people living in these medically underserved areas will have higher preventable ED use [13, 14]. However, finding 
underserved areas is not easily available by using only SD modeling.

Through merging SD and GIS methods, we can integrate demographic data with other datasets outside of the health system such as census data and American FactFinder survey results. Therefore, we will progress the Institute of Health Triple Aim that is improving population health, reducing health care costs, and increasing the quality of care [15]; as Beck et al. [16] explain how different sources of data can be integrated together to have a better understanding of health disparities. Contrary to the majority of these studies that have illustrated the static geographical data using the GIS software, we illustrate our dynamic SD simulation results over the time. Hence, we will reveal insights that were not available merely through GIS mapping.

System dynamicists have strived to combine SD with GIS methodology such as the work by Hovmand and Pitner [17] which is a combination of SD, GIS, and social networks, or the study by Zhan et al. [18] that is a blend of SD with dynamic simulation. As well, Neuwirth et al. [19] explain different spatial SD models and group them into three different categories which are local processes, diffusion processes, and processes that are changing the underlying spatial structure. Our approach is close to local processes since we have ignored the spatial neighborhood interactions between variables, however, we are not bounding stocks and flows to spatial locations, and we are using the results of the SD model to illustrate on the map. In addition, unlike Hovmand and Pitner [17], we are comparing variables associated with different geographic locations, which addresses the complexities of considering spatial factors in our analysis. To the best of our knowledge, the most related work to our study, from a methodological perspective, is the research done by $\mathrm{Xu}$ and Coors [20] in which the authors combined SD and GIS to study the urban residential development. According to them, this kind of methodology integration enables the researchers to explain the variables variation in more details. Also from a conceptual perspective, our work is close to the study done by Brown et al. [21], who measured the ratio of adults per PC providers and highlighted the regions with low access to PC.

In this project, we integrate SD with GIS methods to study a health care issue focusing on access to PC services. We compare the variables associated with each geographic region and their influence on the final behavior of the system, whereas current studies such as Zhan et al. [18] and $\mathrm{Xu}$ and Coors [20], do not have this kind of comparisons, and they are not considering different geographic locations with different spatial characteristics. In addition, our proposed choropleth
GIS map, unlike previous studies including Brown et al. [21], is dynamic and demonstrates the Medicaid enrollees growth throughout several counties in New York State from 2008 to 2020. In this way, we are not just looking at the feedback structure of the system, but we are also adding spatial analysis to our results. In addition, our work, compare to previous studies, is computationally less intensive in studying the dynamics and temporal dimensions of the system.

The remainder of this paper is set as follows. We explain the method and its principles in detail by providing a simple algorithm. We propose our case study with its results. Then, we discuss the results and implications. In the end, we mention the limitations and future ideas.

\section{Methods}

In our methodology, we combine the simulation results from Vensim software with ArcGIS software and we propose an algorithm that produces a GIS map that is dynamic over time and space. In this way, we are capable of identifying hot spots through the region that need health interventions. Researchers have used other integration techniques to develop spatial SD models as well. Scheffran et al. [22] combined STELLA with ArcToolbox GIS software, Mazzoleni et al. [23] integrated SIMILE and ArcView, Lowry and Taylor [24] merged Powersim with Google Earth, Gharib [25] used object-oriented programming to facilitate the interface between Vensim and ArcGIS, and Singhasaneh et al. [26] applied older techniques like combining STELLA with SuperCard. However, we believe our new approach is capable of explaining the static spatial information within a dynamic simulation model in a computationally less intensive way.

In order to combine our SD model with ArcGIS software to study spatial factors, we mainly followed the bellow algorithm consisting of four steps. This process would be the same for any other simulation techniques; therefore, modelers can combine their models developed with other SD software with ArcGIS by following the same steps of the algorithm. Since we merely aim to explain the process of combining these two methodologies, one needs to learn how to work with ArcGIS software through available sources before running the algorithm.

\subsection{Step 1 - determining the geographic boundary and spatial variables}

Before starting the modeling process, we need to determine the geographic boundary of your model. It 
can be a state, a county, or a zip-code area. After developing the simulation model and validating that based on the proposed tests [27], we need to identify the variables that vary spatially and are of particular interest for illustration purposes. In addition, we might be interested in comparing these variables with demographic variables of each area such as income level or educational level. These kind of variables are available in American FactFinder and are called the Census Bureau's Demographic Data which come from different censuses and surveys [28].

\subsection{Step 2 - fetching the Shapefiles and demographic factors}

Now, we need to get the TIGER/Line Shapefile of the geographical area that our model is producing results. A shapefile is a geospatial data format that illustrates vector data such as lines, points, and polygons. We can fetch the Shapefile of those counties/states from Census [29]. In addition, we can download the location of roads, lakes, or any other geographical environments that are important in our analysis, from Census. Census is mainly reporting detailed data in tracts level; therefore, if our model is based on zip code or state level, we need to convert zip-code data to the tract level or we need to assign zip codes and appropriate proportions to corresponding tracts. We will explain this process later in our case study.

\subsection{Step 3 - mapping the simulation results}

In this step, we need to link the simulation results to the Shapefiles. Once we retrieve the Shapefiles and insert them into ArcGIS software, we need to map the variables chosen in the first step. Each Shapefile has a geographic entity code and we should allocate them to the chosen spatial variables to illustrate the corresponding data with each area. Also, we must meet the ArcGIS software linkage requirements to be able to map the results [30].

\subsection{Step 4 - analyzing the influence of spatial factors on system's behavior}

After illustrating the results and observing the changes of the choropleth map over the course of the simulation period, the differences among the selected variables in step 1 need to be analyzed. The analysis needs to be run for each geographic area in order to understand the influence of spatial factors including distance and location of objects on these variables.

\section{Case study - access to primary care (PC) for Medicaid beneficiaries}

Irshaidat et al. [31] and Sabounchi et al. [32] used SD modeling to investigate the effect of Medicaid expansion and the Affordable Care Act (ACA) on PC access in Sothern Tier Counties in New York State. SD methodology is a deterministic simulation technique, which frames and models a complex issue to assist in understanding its underlying structure by inspecting its components and facilitating communication among stakeholders [27]. SD yields a broader perspective to propose sustainable and effective strategies. Irshaidat et al. [31] captured different stages of PC access including getting qualified for Medicaid, enrolling in Medicaid, seeking PC services, and utilizing PC services. The model has been validated by matching the historical data (see Figure 1) using the Maximum Likelihood Ratio [33] and discussing the results with experts in the field. A snapshot of the model is available in the supplementary material (see Appendix A).

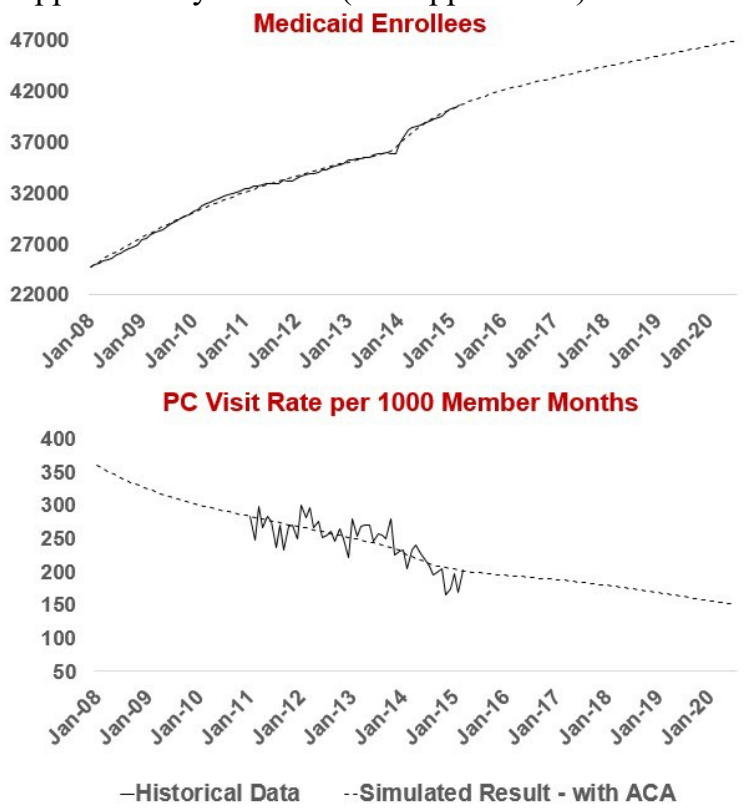

\section{Figure 1 - Simulation results vs. historical data}

The historical trends demonstrate that although Medicaid enrollees have increased, PC visit rate has decreased. However, based on the SD model, we could not understand the reason behind this dynamic. Since spatial factors, such as the distance of providers to residential areas of Medicaid beneficiaries, is a major barrier for access to PC, we hypothesized that spatial variables that are not included in the feedback and 
delay structures of the model are creating the declining trends for PC visit rate. Therefore, we needed a tool that could facilitate the investigation of spatial factors in order to test our claims. Hence, we adopted GIS methods to integrate it with our SD model results to study temporal and spatial variables simultaneously.

\subsection{Step 1 - identifying variables}

We used the simulation model developed by Irshaidat et al. [31] for four different counties in Sothern Tier New York including Broome, Delaware, Tioga, and Chemung in order to feed the GIS map and study the access to PC. In the first step of the algorithm, we identified two variables, 'Number of Medicaid Enrollees' and 'Primary Care Visit Rate' from the model for mapping purposes. In addition, we used the providers' location who had PC visits to illustrate on the map. We got the addresses from the Delivery System Reform Incentive Payment (DSRIP) dashboard and then geocoded them for illustration purposes by using ArcGIS software. We do not explain the geocoding process which is discussed extensively in these references $[34,35]$.

\subsection{Step 2 - fetching}

We fetched the Shapefiles of Broome, Delaware, Tioga and Chemung counties and their major roads from Census [29].

The simulation model results vary at the county level, however, we wanted to illustrate them at the Census tracts level. Therefore, we used the number of Medicaid enrollees in each Census tract available from American FactFinder [28] to obtain the percentages of Medicaid enrollees living in each census tract in 2015. Then we distributed the simulated results for the number of Medicaid enrollees to census tracts (see Appendix B).

In addition, the values of $\mathrm{PC}$ visit rate are simulated at the county level. In order to find the simulated PC visit rate for each census tract, we used DSRIP data for PC visit rate at the zip code level and converted it to the tract level based on the Office of Policy Development and Research guidelines [36]. The details of this conversion process are discussed in Appendix C. Then, using the percentage of PC visit rate for each tract, we distributed the simulated PC visit rate among the tracts of each county based on the process discussed in Appendix B.

\subsection{Step 3 - results}

We chose the number of Medicaid enrollees and PC visit rate for creating the GIS maps for the four counties. In Figure 2, Medicaid enrollees trend in Broome County is visualized in five different color shades. The darker, the more Medicaid beneficiaries are living in those census tracts. We also demonstrate the locations of PC providers on the map by geocoding all the addresses for different PC providers including nurse practitioners, doctor of medicines, practitioners, and registered nurses who accepted Medicaid patient for a PC visit. We also included the interstates and states roads on the map for better visualization.

Since the simulation model runs from 2008 to 2020 on a monthly basis, a snapshot of the dynamic GIS map for January 2008 is shown on the left panel of Figure 2 and January 2020 on the right panel. Based on the simulation results, the number of Medicaid enrollees are increasing throughout the county and so the Broome County map's color is darker in 2020.

Some underserved regions throughout Broome County are clearly visible on the map. For instance, on the right upper side of the map, no PC providers are practicing in Harpursville, which is one of the poorest areas in the county. Therefore, Medicaid enrollees living in this area need to travel a long distance to get to a PC provider. The same situation for people living in Whitney Point can be seen on the left upper side of the map. There are only three PC providers practicing in this area. On the other hand, Vestal, which can be seen in the enlarged box, is the richest region in the county and among the wealthiest in Southern Tier Counties. It has only one-sixth of the total number of Medicaid enrollees comparing to total Medicaid enrollees living in Harpursville. However, the majority of the PC providers are practicing in this wealthy area. This map clearly shows the hot spots and maldistribution of providers in Broome County. The reasons for this discrepancy are hidden to us, but policymakers definitely need to target these hot spots for establishing PC centers.

We also illustrate the $\mathrm{PC}$ visit rate per 1000 members throughout the county in Figure 3 by five different intervals. The lighter color shows lower PC visit rate in the census tract. When we compare the PC visit rate for poor regions like Harpursville that have a high number of Medicaid enrollees, we realize that these areas have the lowest PC visit rate. More surprising is that these rates are almost the same as wealthier regions like Vestal. Moreover, when we compare the PC visit rate in Broome County in 2008 and 2020, we realize the sharp decline in the PC visit rate throughout the county. The dynamic GIS maps provide clear evidence for the reason of the decline in utilization of PC services, which is the lack of access 
to $\mathrm{PC}$ in areas with high needs and geographical maldistribution of providers.

We also expand the same analysis to other three counties in Southern Tier and show the number of Medicaid enrollees and PC visit rate for Chemung, Tioga, Broome, and Delaware County from left to right In Figure 4 and Figure 5. The number of
Medicaid enrollees are increasing significantly throughout these counties between 2008 and 2020 . However, we still see the same problem, which is the lack of access to PC providers in rural areas with a higher number of Medicaid beneficiaries, and consequently a low PC visit rate for these regions (See Figure 6 and Figure 7).

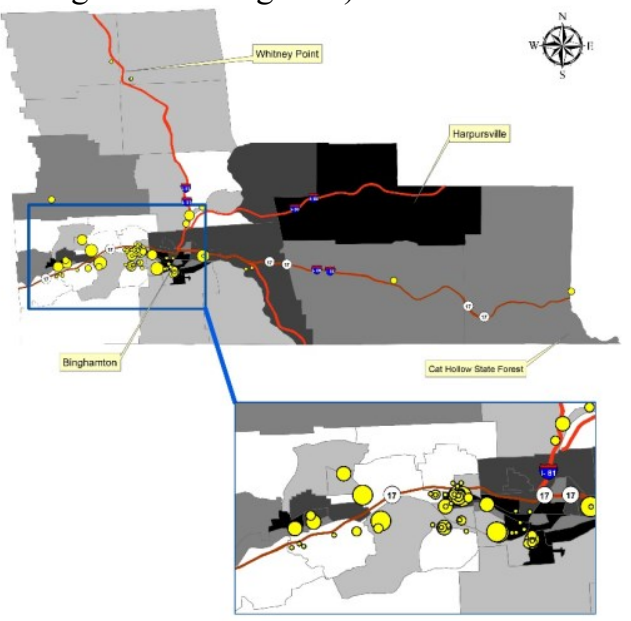

Figure 2 - Medicaid enrollees in Broome County - we have Whitney Point on upper left, Harpursville on upper right, Vestal in lower left, and Cat Hollow State Forest on lower right. [32]
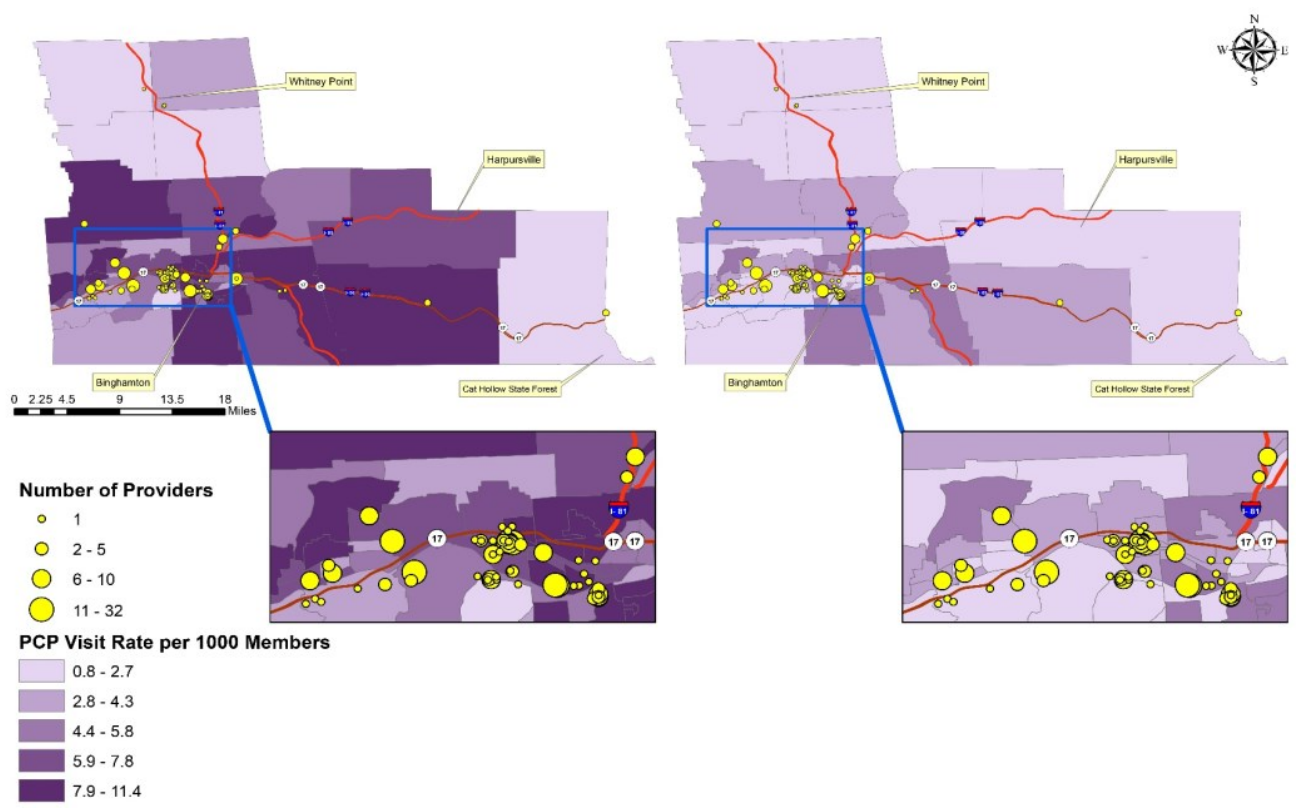

Figure 3 - Primary care visit rate in Broome County [32] 


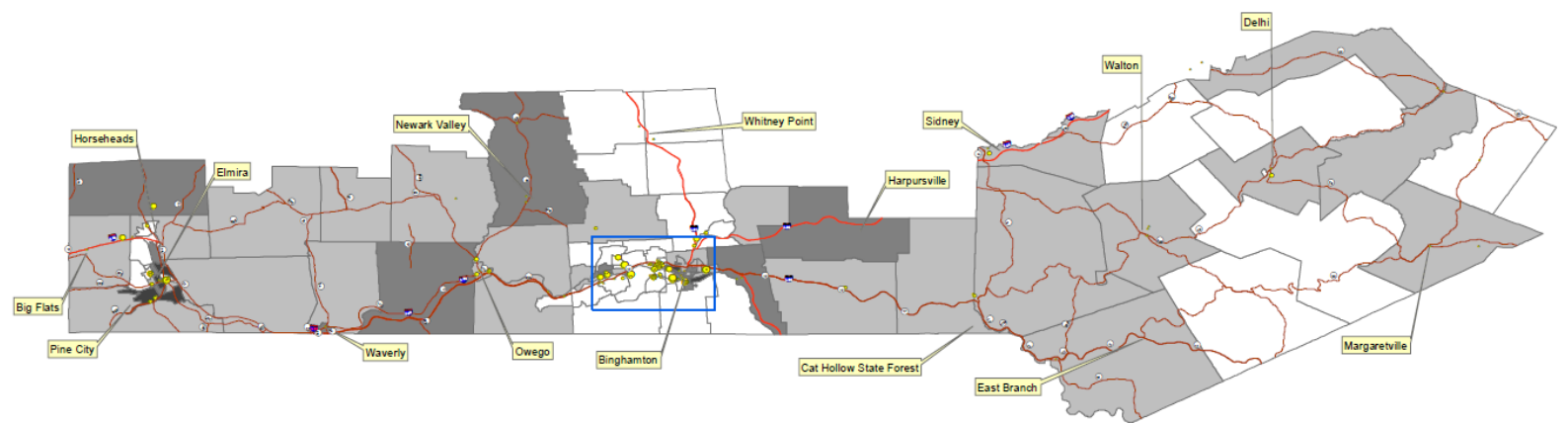

Figure 4 - Medicaid enrollees in Southern Tier Counties in 2008 - the counties from left to right are Chemung, Tioga, Broome, and Delaware.

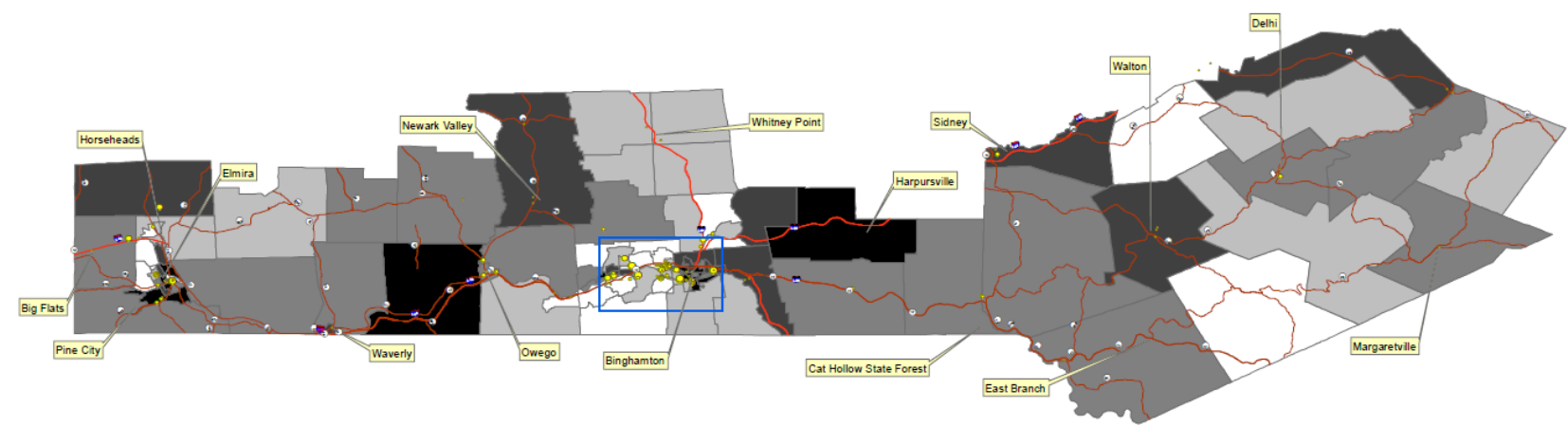

Figure 5 - Medicaid enrollees in Southern Tier Counties in 2020

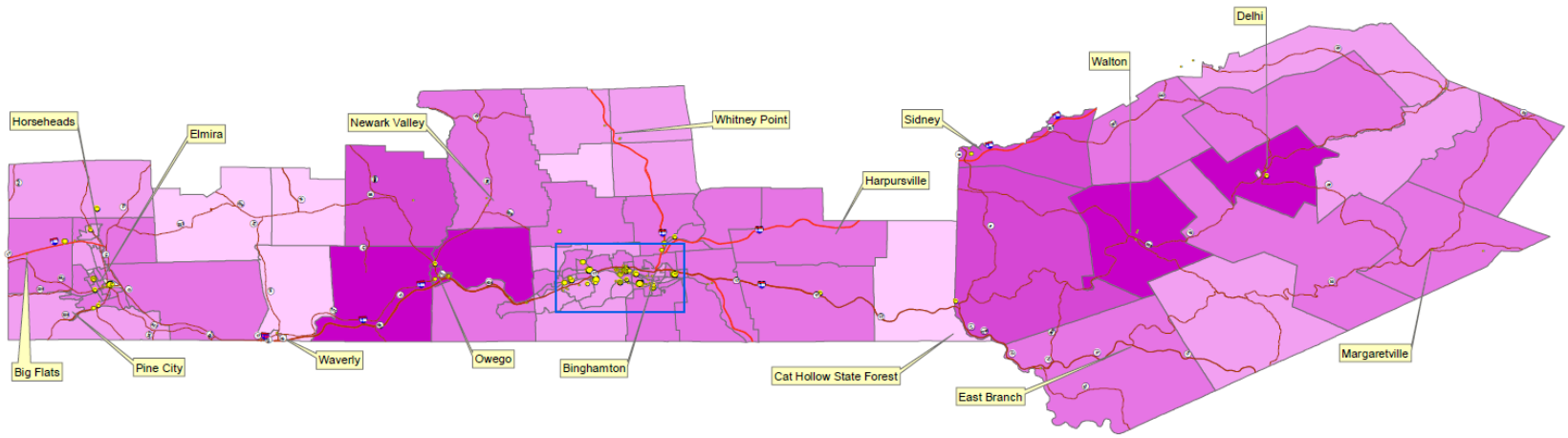

Figure 6 - Primary care visit rate in Southern Tier Counties in 2008

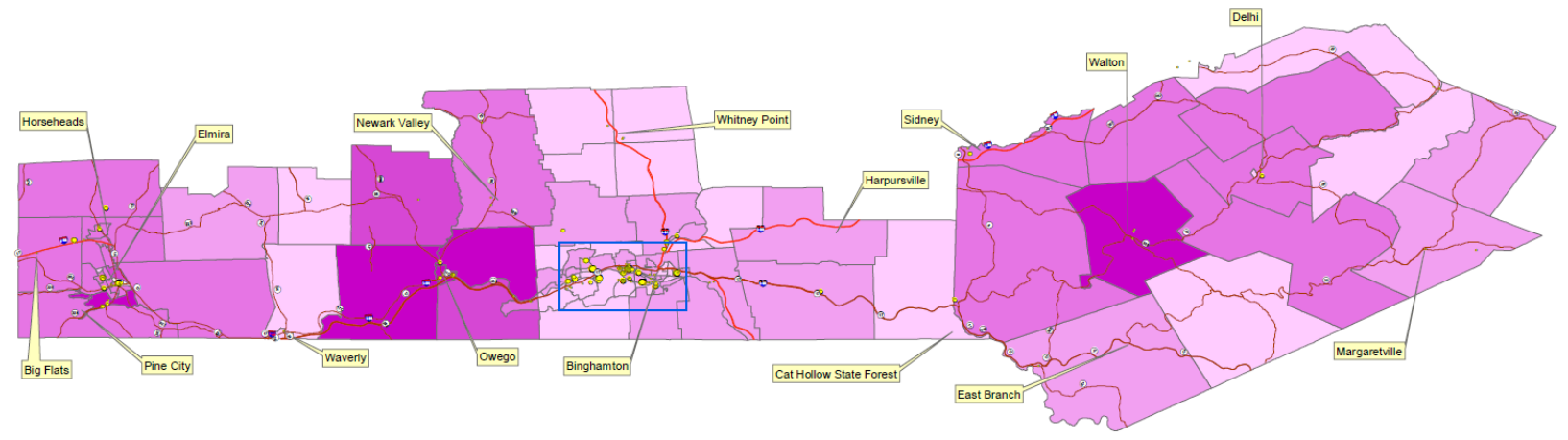

Figure 7 - Primary care visit rate in Southern Tier Counties in 2020 


\subsection{Step 4 - discussion}

The parameters calibrated in the simulation model by Irshaidat et al. [31] are listed in Table 1. We use these parameters to compare spatial differences among the four counties. In other words, we are not just studying the influence of feedback structure on the final behavior of the system, but also expanding the SD simulation modeling and analysis by considering the spatial factors. Therefore, comparing different parameters' values from these four counties would reveal information related to access to care issues and spatial characteristics of each county.

According to Table 1, the average time it takes for a Medicaid patient to get an appointment and make the visit to PC provider's office is lowest in Chemung County compared to other counties. It emphasizes the better access or lower perceived barriers among Medicaid enrollees in this county. However, Medicaid patients in Chemung County delay their decision to continue or quit PC by 26 days that is highest compared to other counties.

In Delaware County, the average time to decide to stay in preventive care is lowest, almost 9 days, which emphasizes that Medicaid patients would decide much faster whether to continue or quit. However, it is most probable that they would never comply again since the 'Fraction never complying again' is $51.2 \%$ in this county. This indicates that Medicaid patients faced many barriers during the first visit and it made the decision easier for them to quit utilizing PC services.

Table 1 - Calibrated parameters in SD model

\begin{tabular}{|c|c|c|c|c|c|}
\hline Parameters & Definitions & Chemung & Tioga & Broome & Delaware \\
\hline $\begin{array}{l}\text { Average Time to } \\
\text { Overcoming } \\
\text { Barriers (Days) }\end{array}$ & $\begin{array}{l}\text { The average time it takes for a Medicaid patient, } \\
\text { once decided to utilize care to get an appointment } \\
\text { and make the visit to the PCP office. }\end{array}$ & 0.83 & 58.69 & 25.39 & 14.30 \\
\hline $\begin{array}{l}\text { Average Time to } \\
\text { Decide Whether } \\
\text { Stay in } \\
\text { Preventative Care } \\
\text { (Days) }\end{array}$ & $\begin{array}{l}\text { The average time it takes for a Medicaid patient } \\
\text { once made the visit to PCP office to choose to } \\
\text { continue utilizing preventive care, or to quit ever } \\
\text { going to a PCP, or delay utilizing preventive } \\
\text { services to a future point in time. }\end{array}$ & 26.38 & 20.28 & 17.21 & 8.76 \\
\hline $\begin{array}{l}\text { Fraction always } \\
\text { complying }\end{array}$ & $\begin{array}{l}\text { The fraction of Medicaid Enrollees who started to } \\
\text { utilize care, who decided to continue utilizing } \\
\text { preventive care on a permanent basis. }\end{array}$ & $33.4 \%$ & $22.8 \%$ & $14.8 \%$ & $12.2 \%$ \\
\hline $\begin{array}{l}\text { Fraction never } \\
\text { complying again }\end{array}$ & $\begin{array}{l}\text { The fraction of Medicaid Enrollees who started to } \\
\text { utilize care, who decided to never utilize } \\
\text { preventive care in the future. }\end{array}$ & $35.1 \%$ & $54.3 \%$ & $68.7 \%$ & $51.2 \%$ \\
\hline $\begin{array}{l}\text { Fraction flowing } \\
\text { back }\end{array}$ & $\begin{array}{l}\text { The fraction of Medicaid Enrollees who started to } \\
\text { utilize care but decide to delay utilizing } \\
\text { preventive care at a later time. }\end{array}$ & $31.4 \%$ & $22.8 \%$ & $16.5 \%$ & $36.6 \%$ \\
\hline
\end{tabular}

It seems that easier access to PC would make patients delay their decisions to quit or continue utilizing PC services. The reason is that when patients can get to the provider's office easier, they recognize that it will not be difficult to return for a second or third visit. Thus, they will establish a regular PC visit with a longer delay. Nevertheless, if patients experience more barriers in their first visit to the provider's office, a higher fraction of them would quit the PC. These barriers seem more significant in larger counties like Broome and Delaware because it takes more time until Medicaid enrollees overcome their barriers to access care, which is 25 and 14 days respectively. Even when they do, it takes them a shorter time to decide whether to stay in PC since they faced many barriers in getting to the provider's office. Thus, they recognize that they need to overcome the same barriers to make a return visit and consequently decide not to comply with utilizing PC services on a routine basis. In conclusion, the barriers in accessing PC provider's locations including transportation issues and distance to the providers are the leading factors in Medicaid enrollees' decisions to continue utilizing PC services.

Table 2 shows the demographic characteristics of each county. The number of providers has a positive correlation with Medicaid enrollees in each county $(\mathrm{r}=99 \%)$. However, Delaware County, which is the poorest and largest county among these counties, has the lowest number of providers. Although Delaware County has the smallest population and so requires fewer PC providers, the majority of providers are located in urban areas of the county. Therefore, Medicaid enrollees who are residing in rural areas of the county, experience the most difficulty in accessing care.

On the other hand, Tioga County is the wealthiest county based on median household income and per capita income (See Table 2) but has almost the same 
number of Medicaid enrollees and providers as Delaware County. Since Tioga County is much smaller than Delaware County, so the geographical maldistribution of providers throughout this county might not lead to the same level of difficulties for access to $\mathrm{PC}$ for patients in rural areas comparing to Delaware. Moreover, Broome County has the highest ratio of providers per Medicaid enrollee, but due to noticeable maldistribution of providers in this county, $\mathrm{PC}$ visit rate is declining even with increasing number of Medicaid Enrollees.
In conclusion, the proper geographical distribution of PC providers should be our first priority in larger and poorer counties in order to facilitate the access to care for Medicaid population.

Table 2 - Demographic characteristics of counties

\begin{tabular}{lcccc}
\hline Factor/County & Chemung & Tioga & Broome & Delaware \\
\hline Population (2015) & 87,071 & 49,453 & 196,567 & 46,053 \\
Medicaid enrollees (2015) & 17718 & 8494 & 40204 & 8413 \\
Ratio of Medicaid enrollees to population (2015) & 0.203 & 0.171 & 0.204 & 0.182 \\
National Average of this ratio (2015) & & & 0.218 & \\
Number of providers who had primary care visits & 74 & 19 & 275 & 20 \\
Providers per Medicaid enrollee & 0.0042 & 0.0022 & 0.0068 & 0.0024 \\
Area & 407 & 518 & 705 & 1442 \\
Per capita income & $\$ 26,262$ & $\$ 29,427$ & $\$ 25,105$ & $\$ 23,835$ \\
Median household income & $\$ 50,320$ & $\$ 57,514$ & $\$ 46,261$ & $\$ 43,720$ \\
\hline
\end{tabular}

\section{Implications}

In the absence of spatial factors within SD models, we integrate SD with GIS methods to study the influence of these factors on the dynamic behavior of the system and the interaction of spatial and systemic variables within the system. We developed the first dynamic GIS map based on the SD simulation results for a healthcare issue. In our approach, we integrate data from multiple sources that would help us to assess the needs of different regions and propose interventions to improve the health of communities [37].

Our approach provides a decision support tool for policymakers to identify demographic regions that are in strong need of PC providers. Hence, they will target these regions to establish $\mathrm{PC}$ settings or to remove barriers for patients in accessing care. Overall, this tool would facilitate the communications among stakeholders to design effective interventions that would improve access to PC considering spatial and temporal characteristics of the region. In addition, our work presents a framework that can be used by researchers to integrate simulation models with GIS methods and study spatial factors in their analysis in a very straightforward process.

\section{Limitations and Future works}

This work has a number of limitation that can be addressed by gathering more geographic and demographic data. For instance, the number of providers who had PC visits was just available for one year, 2015, through DSRIP dashboard. Hence, we assumed the changes in the number of PC providers and its influence on PCP visit rate over the simulation period has been minimal. We did not investigate the effect of proximity of other providers practicing in areas above Southern Tier Counties on access to PC for Medicaid enrollees. In addition, we identified hot spots, however, more strategic analyses are needed in order to design and propose applicable public health interventions to improve the health outcomes of Medicaid beneficiaries, because there are no regulations forcing providers to practice in rural and underserved areas.

To expand our analysis, we are restructuring our simulation model to use claims data for modeling and validation purposes. We will consider Medicaid superutilizers in Utah State counties and study the influence of behavioral, spatial, and systemic factors on users and various health outcomes within each geographic location. Then we will combine the simulation model with GIS methods to investigate the effects of spatial variables such as proximity to providers and emergency departments, and demographic variables on PC utilization dynamic 


\section{References}

[1] Ford, A. and F.A. Ford, Modeling the environment: an introduction to system dynamics models of environmental systems. 1999: Island press. Available from

[2] Song, S.J. and R.M. M'gonigle, Science, power, and system dynamics: the political economy of conservation biology. Conservation Biology, 2001. 15(4): p. 980-989.

[3] Lyneis, J.M. and D.N. Ford, System dynamics applied to project management: a survey, assessment, and directions for future research. System Dynamics Review: The Journal of the System Dynamics Society, 2007. 23(23): p. 157-189.

[4] Sterman, J., R. Oliva, K.W. Linderman, and E. Bendoly, System dynamics perspectives and modeling opportunities for research in operations management. 2015.

[5] Sharareh, N., N.S. Sabounchi, A. Roome, R. Spathis, and R.M. Garruto, Model-based risk assessment and public health analysis to prevent Lyme disease. Royal Society open science, 2017. 4(11): p. 170841.

[6] Sharareh, N., N.S. Sabounchi, H. Sayama, and R. MacDonald, The Ebola Crisis and the Corresponding Public Behavior: A System Dynamics Approach. PLoS currents, 2016. 8.

[7] Tian, Y., K. Govindan, and Q. Zhu, A system dynamics model based on evolutionary game theory for green supply chain management diffusion among Chinese manufacturers. Journal of Cleaner Production, 2014. 80: p. 96-105.

[8] Dulin, M.F., T.M. Ludden, H. Tapp, J. Blackwell, B.U. de Hernandez, H.A. Smith, et al., Using Geographic Information Systems (GIS) to understand a community's primary care needs. The Journal of the American Board of Family Medicine, 2010. 23(1): p. 13-21.

[9] Edward, J. and D.J. Biddle, Using geographic information systems (GIS) to examine barriers to healthcare access for Hispanic and Latino immigrants in the US south. Journal of racial and ethnic health disparities, 2016: p. 1-11.

[10] Garcia, E., N. Serban, J. Swann, and A. Fitzpatrick, The effect of geographic access on severe health outcomes for pediatric asthma. Journal of Allergy and Clinical Immunology, 2015. 136(3): p. 610-618.

[11] McLafferty, S. and S. Grady, Prenatal care need and access: a GIS analysis. Journal of medical systems, 2004. 28(3): p. 321-333.

[12] Rosero-Bixby, L., Spatial access to health care in Costa Rica and its equity: a GIS-based study. Social Science \& Medicine, 2004. 58(7): p. 1271-1284.

[13] Fishman, J., S. McLafferty, and W. Galanter, Does Spatial Access to Primary Care Affect Emergency Department Utilization for Nonemergent Conditions? Health Services Research, 2016.

[14] Okunseri, C., R. Vanevenhoven, T. Chelius, K.M. Beyer, E. Okunseri, W.K. Lobb, et al., Travel distances by
Wisconsin Medicaid enrollees who visit emergency departments for dental care. Journal of public health dentistry, 2016.

[15] Miranda, M.L., J. Ferranti, B. Strauss, B. Neelon, and R.M. Califf, Geographic health information systems: a platform to support the 'triple aim'. Health Affairs, 2013. 32(9): p. 1608-1615.

[16] Beck, A.F., M.T. Sandel, P.H. Ryan, and R.S. Kahn, Mapping Neighborhood Health Geomarkers To Clinical Care Decisions To Promote Equity In Child Health. Health Affairs, 2017. 36(6): p. 999-1005.

[17] Hovmand, P.S. and P. Pitner, Combining System Dynamics, Social Networks, and Geographic Information Systems. Information Systems, 2014.

[18] Zhan, J., X. Deng, Q. Jiang, and N. Shi. The application of system dynamics and CLUE-S model in land use change dynamic simulation: A case study in Taips County, Inner Mongolia of China. in Proceedings of the 2007 Conference on Systems Science, Management Science and System Dynamics: Sustainable Development and Complex Systems. 2007.

[19] Neuwirth, C., B. Hofer, and A. Peck, Spatiotemporal processes and their implementation in Spatial System Dynamics models. Journal of Spatial Science, 2015. 60(2): p. $277-288$.

[20] Xu, Z. and V. Coors, Combining system dynamics model, GIS and 3D visualization in sustainability assessment of urban residential development. Building and Environment, 2012. 47: p. 272-287.

[21] Brown, E.J., D. Polsky, C.M. Barbu, J.W. Seymour, and D. Grande, Racial disparities in geographic access to primary care in Philadelphia. Health Affairs, 2016. 35(8): p. 1374-1381.

[22] Scheffran, J., T. BenDor, Y. Wang, and B. Hannon. A spatial-dynamic model of bioenergy crop introduction in Illinois. in International Conference of System Dynamics Society. 2007. Citeseer.

[23] Mazzoleni, S., F. Giannino, M. Colandrea, M. Nicolazzo, and J. Massheder, Integration of system dynamics models and geographic information systems. Simile, 2003. 600(5)

[24] Lowry, T. and R. Taylor. Visualizing spatial and temporal dynamics in Google Earth using Powersim. in 27th International Conference of the System Dynamics Society, Albuquerque. 2009.

[25] Gharib, S. (2008). Synthesizing system dynamics and geographic information systems in a new method to model and simulate environmental systems. Thesis. The University of Bergen. Retrieved from http://bora.uib.no/handle/1956/3296

[26] Singhasaneh, P., J.E. Lukens, A. Eiumnoh, and H. Demaine. Integration of Raster-Based GIS and System Dynamics and Its Application. in International System Dynamics Conference, Bangkok, Thailand. 1991. 
International System Dynamics Conference, Bangkok, Thailand.

[27] Sterman, J.D., Business dynamics: systems thinking and modeling for a complex world. 2000. Available from

[28] Census. American FactFinder. 2017 [cited 2017;

Available from:

https://factfinder.census.gov/faces/nav/jsf/pages/index.xht $\mathrm{ml}$.

[29] Census. Maps \& Data. 2017 [cited 2017; Available from: https://www.census.gov/geo/maps-data/.

[30] IEGS. Joining Census Data Tables to Shapefiles in ArcMap. 2013 [cited 2018; Available from:

http://spatial.scholarslab.org/stepbystep/joining-censusdata-tables-to-shapefiles-in-arcmap/.

[31] Irshaidat, F., N. S. Sabounchi, and A.S. Atav. (2016). Three essays on advancing the system dynamics modeling approach for analyzing policies and interventions for improvement of women's health. Thesis. State University of New York at Binghamton.

[32] Sabounchi, N., N. Sharareh, F. Irshaidat, and S. Atav, Spatial dynamics of access to primary care for the medicaid population. Health Systems, 2018: p. 1-12.

[33] Dogan, G. Confidence interval estimation in system dynamics models: Bootstrapping vs. likelyhood ration method. in 22nd International Conference of the System Dynamics Society. Oxford, UK. 2004.

[34] Kurland, K.S. and W.L. Gorr, GIS tutorial for health. 2007: ESRI, Inc. Available from

[35] ESRI. Geocoding a table of addresses in ArcMap. 2016 [cited 2017; Available from:

http://desktop.arcgis.com/en/arcmap/10.3/guidebooks/geocoding/geocoding-a-table-of-addresses-inarcmap.htm.

[36] PD\&R. HUD-USPS Crosswalk Files. 2017 [cited

2017; Available from:

https://www.huduser.gov/portal/datasets/usps_crosswalk.ht ml\#data.

[37] Sage, W.M., M. Balthazar, S. Kelder, S. Millea, S. Pont, and M. Rao, Mapping data shape community responses to childhood obesity. Health Affairs, 2010. 29(3): p. 498-502. 